\title{
Effects of Pelvic Stability Training on Movement Control, Hip Muscles Strength, Walking Speed and Daily Activities after Stroke: A Randomized Controlled Trial
}

\author{
Lavnika Dubey Suruliraj Karthikbabu Divya Mohan \\ Department of Physiotherapy, School of Allied Health Sciences (SOAHS), Bangalore Campus, Manipal University, \\ Bangalore, India
}

\author{
Keywords \\ Stroke $\cdot$ Pelvic stability $\cdot$ Mobility $\cdot$ Strength $\cdot$ Movement \\ control
}

\begin{abstract}
Background: Pelvic instability is a common occurrence during standing and walking post stroke. Inappropriate muscle activation and poor movement control around the pelvis lead to difficulty in mobility and daily functioning. Purpose: The purpose of the study was to examine the effects of pelvic stability training on the movement performance of trunk and lower limb, hip muscles strength, walking speed and daily activities after stroke. Methods: This randomized controlled trial involved 34 patients aged $56(11)$ years with the post stroke duration being 31 (22) weeks. Patients with medical stability, an ability to follow verbal instructions, independent sitting balance and supervised walking capacity were included. The experimental group $(n=13)$ underwent the pelvic stability training and the control group $(n=13)$ were subjected to standard physiotherapy for $1 \mathrm{~h}$ a day, 3 times a week for over 6 weeks in a stroke rehabilitation setting. Trunk
\end{abstract}

\section{KARGER}

(c) 2018 S. Karger AG, Basel

E-Mail karger@karger.com

www.karger.com/aon
Impairment Scale 2.0 (TIS 2.0), Fugl Meyer Assessment of Lower Extremity (FMA-LE), hip muscles strength (lb.), gait speed, pelvic tilt and modified Barthel Index (MBI) were the outcome measures. Results: All the measures between both groups were similar at baseline except TIS 2.0 and FMA-LE. Following training, pelvic stability group showed statistically significant improvement $(p<0.05)$ except MBI and substantial mean changes in the measures of TIS 2.0 (2.12), FMALE (5.12), hip strength (lb.) for flexors (4.6), extensors (2.8), abductors (2.58), adductors (2.9), gait speed $(0.05 \mathrm{~m} / \mathrm{s})$ and MBI (7.74) as against standard physiotherapy group. Conclusion: Pelvic stability training was found to be beneficial in improving the trunk and lower extremity movement control, hip muscles strength, gait speed and daily activities in stroke.

(c) 2018 S. Karger AG, Basel

\section{Introduction}

About two-third of patients after stroke showed poor walking capacity [1] and $72 \%$ of them demonstrated severe lower limb motor deficits during sub-acute and 
chronic stage stroke recovery [2]. Following stroke, an inappropriate muscular activity pattern associated with spasticity and weakness, soft tissue stiffness of lower limb and postural deviations of trunk and pelvis might affect the postural control system as a whole [3]. Pelvis being the key structure that connects the trunk to lower extremities supports the weight of the body and transfers its load onto lower limbs. Also, pelvis is a part of the lower trunk when an individual is in the sitting position, whereas it becomes a functional component of the lower limb when a person is standing and walking.

Pelvic stability refers to the ability of coordinated activity between the lower trunk and proximal hip muscles during functional balance and mobility tasks in which the pelvis serves the proximal dynamic stability as to allow for effective lower limb mobility [4]. In a recent cross-sectional study, it was observed that poor trunk control and lower extremity recovery are related to the pelvis instability in patients with chronic stroke [5]. Altered pelvic alignment in the standing position resulting from poor lower trunk control after stroke shall positively influence the balance, gait and functional performance [6]. Additionally, it was found that pelvic mal-alignment, that is, excessive lateral and anterior pelvic tilt shall affect their standing weight-bearing symmetry between feet in patients after stroke [7]. While walking after stroke, poor lower trunk control and pelvic instability might allow for excessive lateral pelvic displacement towards the least involved side and reduced vertical movement on the most affected side [8].

Postural control in stroke is affected not only by the most involved lower limb but also by the inability of contralateral leg to compensate for the undue postural demand from paretic leg [9]. In response to self-initiated perturbations in the standing posture after stroke, there was a reduced gluteus medius muscular activity on the most involved side with an exaggerated activity of hip adductors on the least involved side [10]. An enhanced hip extensor activity of either the most affected or least affected leg in the stance phase of gait was found to be positively correlated with the walking speed [11]. Hip muscles particularly the extensors and adductors are the weakest in patients with chronic stroke and they are related to worse gait symmetry [12]. Hip extensor muscle weakness and restricted hip extension mobility on the most involved lower limb during the terminal stance of gait shall invariably influence the movement control of ipsilateral side hip and knee flexion throughout the swing phase, thus reducing the gait speed in stroke [13, 14].

Pelvic Stability Training on Movement

Control and Daily Activities after Stroke
Although pelvic stability is an integral component for balance and mobility in patients with stroke, the exercise training provided in this regard is till date underexplored. Hence, we attempted to examine the effects of pelvic stability training, that is, the dynamic coactivity and strengthening of lower trunk and proximal hip muscles on trunk and lower extremity movement control, hip muscles strength, walking speed and daily functioning in patients with stroke.

\section{Material and Methods}

The study protocol was approved by the institutional research committee of School of Allied Health Sciences, Manipal University and registered in clinical trial registry (CTRI/2017/04/008309). A written informed consent and details of study participants were obtained from the legally acceptable representatives in their native language. This randomized controlled trial was conducted in the stroke rehabilitation centre of the Manipal hospital. Patients post stroke with medical stability were screened for the study eligibility. Patients with first episode of either haemorrhagic or ischaemic stroke, ability to understand simple verbal commands, standing ability with or without manual assistance/mobility aids, Brunnstrom stage beyond 3 for lower limb motor recovery were recruited in the study. Patients were not included in the study if they had any other neurological and musculoskeletal dysfunction such as cerebellar lesion, perceptual dysfunction and any history of lower limb or pelvic fractures in the previous 6 months that might potentially affect their performance of balance and walking. They were then assigned into either the experimental group (pelvic stability training) or control group (standard physiotherapy) through the block randomization method with concealed allocation using opaque sealed envelopes. Patients from both groups were given $1 \mathrm{~h}$ treatment session per day, 3 times a week for over 6 weeks by qualified physiotherapists. The standard physiotherapy involved $30 \mathrm{~min}$ of a range of motion exercises, tone modulation strategies, stretching, synergy activity and strengthening of lower limb muscles in supine and sitting positions addressing the soft tissue stiffness, spasticity, muscle inactivity and weakness of the lower limb. Balance training in sitting and standing and gait training were administered to them for 30 min duration. The similarity of groups with respect to other treatments was ensured. All the study patients involved only in the allocated exercise regime and they were advised to continue medications as prescribed by a neurologist. During the study period, none of them underwent treatments such as Ayurveda, and massage therapy, which might potentially influence their rehabilitation outcomes.

\section{Pelvic Stability Training}

This exercise protocol was designed based on the neurophysiological and biomechanical aspects of pelvic stability. The muscular selectivity and co-contraction of lower trunk and proximal hip muscles were achieved through training and reinforced through the therapeutic guidance and irradiation strategies in lying, sitting and standing positions. The exercise sets and intensity were designed based on the performance of the individual 
patient. Exercises such as pelvic bridge, unilateral bridging and pelvic rotations in supine positions emphasized the lower trunk abdominals and proximal hip muscular co-activity, particularly the gluteus medius and maximus. These exercises were initially given in plinth and later on using physio ball. The gluteus medius was activated when the patient was lying on the most involved side with both hips and knees flexed to $90^{\circ}$. The patient was instructed to abduct the top hip against the manual resistance applied at the distal thigh by the therapist. Irradiation from hip abductors of the strong leg was made to overflow to the weak gluteus medius muscle. From the same starting position, the patient was guided to lift both knees towards ceiling and was instructed to hold them for 5-10 s. This might reinforce the activity of lower abdominals along with ipsilateral hip abductors and contralateral hip adductors. Tri-phasic burst of hip extensor and flexor muscle groups was activated when the patient was lying on his least involved side. Hip extensors and flexors being the tonic and phasic muscles, respectively, the extensor was activated isometrically at an inner range, that is, extreme hip extension and flexor were contacted isotonically from an outer to an inner range, that is, towards maximum hip flexion. The pelvic stability training protocol is shown in Appendix 1.

While sitting on an unstable support such as therapads, the pelvic muscular coactivity was enhanced using dynamic weight shifts between buttocks. During anterior-posterior weight shifts, the forward trunk inclination with anterior pelvic tilt was encouraged to activate the gluteus maximus and the lower trunk abdominals. The pelvic stability in walk standing and step standing positions was achieved by dynamic weight shifts through tactile cueing of lower trunk abdominals and gluteus maximus. The exercise was further progressed to stepping sideways in standing with posteriorly tilted pelvis, which involved the coordinated activity between quadratus lumborum, adductor and abductors of the hips. Similar to the side lying exercise, the triphasic burst of hip extensor and flexor was achieved during stepping-up over a small block kept in front. In addition, the hip muscles were progressively strengthened using closed kinetic chain exercise machine.

\section{Outcome Measures}

Trunk Impairment Scale 2.0 (TIS 2.0), an ordinal scale measured the movement coordination of lateral flexion and rotation initiated from upper and lower trunk [15]. Fugl Meyer Assessment-Lower Extremity (FMA-LE) scale with good internal consistency and inter-rater reliability was used to examine the motor performance of the lower extremity post stroke [16, 17]. Handheld dynamometer is a clinically reliable instrument and the strength of hip muscles was tested using the dynamometer in stroke studies $[18,19]$. The isometric strength of hip extensors, flexors, abductors and adductors was measured using the break test in pounds (lb.). An average of 3 trials was considered the actual strength value. These test positions were standardized and are showed in Appendix 2. The angle of lateral and anterior pelvic tilt in standing was measured using the palpation meter, a reliable device to measure pelvic instability in stroke $[5,7,20]$. The gait speed and cadence were calculated after a $10-\mathrm{m}$ walk test [21]. Modified Barthel Index (MBI) assessed the level of functional independence in the activities of daily living post stroke [22]. These outcomes were collected by an independent assessor who was involved in conducting neither the study intervention nor the randomization process.

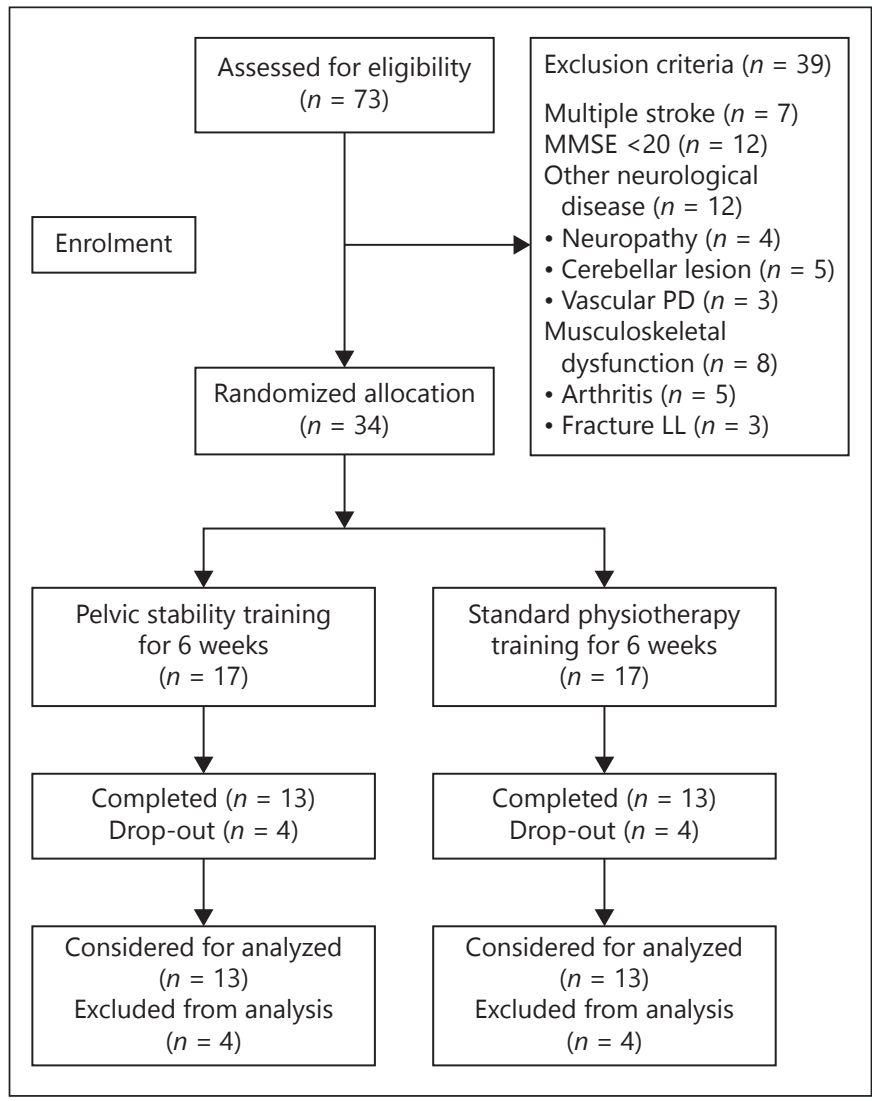

Fig. 1. Flow chart shows the study organization.

\section{Statistical Analysis}

Data was analyzed using the SPSS 16.0 version. Baseline homogeneity of continuous and dichotomous variables between groups was tested using independent $t$ test and chi-square test respectively. We confirmed the equality of variance using Levene's test and therefore conducted the parametric test. Paired $t$ test was used for within-group analysis of outcome measures between preintervention and 6 weeks postintervention levels. The between groups comparison was done using the independent $t$ test. The $p$ value of $<0.05$ was considered statistically significant for the outcome measures. The extent of change in each outcome measure for the individual group was calculated by subtracting the baseline score upon the mean difference between pre- and post-training and the percentage of improvement was reported accordingly. We also identified the relative ratio of progress between 2 groups by dividing the percentage of improvement in standard physiotherapy over pelvic stability training.

\section{Results}

Flowchart depicts the number of patients who were screened, enrolled in the study and those who completed the intervention (Fig. 1). After screening 73 patients for 
Table 1. Demographic characteristics outcome variables at baseline ${ }^{\mathrm{a}}$

\begin{tabular}{lccl}
\hline Variables $^{\mathrm{b}}$ & $\begin{array}{c}\text { Pelvic control } \\
\text { training } \\
(n=17)\end{array}$ & $\begin{array}{l}\text { Conventional } \\
\text { physiotherapy } \\
(n=17)\end{array}$ & $p$ value ${ }^{\mathrm{c}, \mathrm{d}}$ \\
\hline Age, years & $54.35(11.64)$ & $58.24(11.77)$ & 0.34 \\
Post-stroke duration, days & $240(135)$ & $199(176)$ & 0.44 \\
Gender, male/female, $n(\%)$ & $13 / 4(76 / 24)$ & $12 / 5(70 / 30)$ & 0.85 \\
Ischemic/hemorrhagic stroke, $n(\%)$ & $13 / 4(76 / 24)$ & $13 / 4(76 / 24)$ & 1.0 \\
Side affected (right/left), $n(\%)$ & $11 / 6(64 / 36)$ & $13 / 4(76 / 24)$ & 0.68 \\
Fugl meyer assessment - lower limb & $15.71(4.01)$ & $20.53(4.55)$ & 0.003 \\
Hip flexors strength (lb.) & $18.71(6.42)$ & $22.94(4.39)$ & 0.32 \\
Hip extensors strength (lb.) & $19.12(6.07)$ & $23.41(7.05)$ & 0.66 \\
Hip abductors strength (lb.) & $21.41(5.42)$ & $25.18(5.18)$ & 0.06 \\
Hip adductors strength (lb.) & $19.24(6.86)$ & $22.29(6.89)$ & 0.20 \\
Gait speed, m/s & $0.51(0.19)$ & $1.03(1.11)$ & 0.22 \\
Cadence, steps/min & $62.71(16.12)$ & $77.59(20.82)$ & 0.06 \\
Anterior pelvic tilt (degree) & $5.29(1.49)$ & $5.41(1.46)$ & 0.81 \\
Lateral pelvic tilt (degree) & $4.71(1.44)$ & $5.24(1.60)$ & 0.32 \\
Modified Barthel Index & $64.41(18.69)$ & $64.76(19.00)$ & 0.95 \\
Trunk Impairment Scale 2.0 & $4.82(1.23)$ & $8.00(2.15)$ & 0.001 \\
\hline
\end{tabular}

${ }^{\mathrm{a}}$ Values are presented as mean (SD) or number $(\%) ;{ }^{\mathrm{b}}$ ordinal variables analyzed using independent $t$ test; ${ }^{\mathrm{c}}$ nominal variables analyzed using chi square test; ${ }^{\mathrm{d}} p$ value was statistically significant at $<0.05$. study eligibility, 34 patients met the inclusion criteria. Of the 17 patients who were assigned to each treatment group, 13 patients completed the specific intervention protocol. At baseline, the demographic characteristics and outcome variables were similar except FMA-LE ( $p=$ $0.008)$ and TIS $2.0(p=0.001)$ and are presented in Table 1. Seven patients (41\%) from each group had a personal history of either tobacco chewing or cigarette smoking. Nine patients $(53 \%)$ in pelvic stability training group and 10 patients (59\%) in standard physiotherapy group allegedly reported alcohol consumption. Among 34 participants, most of them, 13 patients in each group (76\%) had a previous history of either diabetes mellitus or hypertension or both. The medical report showed that there was an associated cardiac illness in $5(29 \%)$ and $7(41 \%)$ patients from experimental and control groups respectively.

Within-group comparison of all outcome measures post intervention showed statistically significant changes $(p<0.05)$ for both groups. For between-groups comparison, the experimental group, that is, pelvic stability training showed statistically significant improvement in all outcome measures except MBI compared to standard physiotherapy. The corresponding mean changes between groups favor the pelvic stability training group. The mean changes within group and between groups for all outcome measures are showed in Table 2.

Pelvic Stability Training on Movement Control and Daily Activities after Stroke
Among all the outcome measures at the pre-training level, the movement control of trunk in sitting as measured using TIS 2.0 showed threefold changes, that is, the relative ratio of 3.4 and improved the most for the pelvic stability training (77\%) than the standard physiotherapy $(23 \%)$. The pelvic stability training $(44 \%)$ in the FMA-LE measure showed 5 times greater over the standard physiotherapy (8.4\%). The strength (lb.) of hip extensors and adductors in the experimental group (32\%; 33\%) improved 2 times more than that of the control group (15\% each). Thirty five percentage of change in the hip flexor strength following pelvic stability training accounting for greater improvement (ratio of 3.8) compared to $9 \%$ improvement post standard physiotherapy care. Of all strength values, the hip abductors showed lesser changes following pelvic stability (20\%) and standard physiotherapy (8\%) interventions. Nevertheless, the relative ratio (2.6) between 2 groups favors the pelvic stability training. From the baseline walking parameters, the gait velocity and cadence of experimental group improved to 38 and $20 \%$, respectively, whereas it showed just 4 and $6 \%$ changes in the mentioned parameters for the control group. On the pelvic tilt measures, the anterior and lateral pelvic obliquity got corrected by 46 and $56 \%$ in the experimental group as opposed to the control group (28\%; $32 \%)$. The func- 
Table 2. Comparison of outcome measures within and between groups

\begin{tabular}{|c|c|c|c|c|c|c|c|c|}
\hline Outcome variables & \multicolumn{3}{|c|}{ Pelvic stability training* $(n=13)$} & \multicolumn{3}{|c|}{ Standard physiotherapy* $(n=13)$} & \multicolumn{2}{|c|}{ Between group comparison ${ }^{\S}$} \\
\hline FMA-LE & $15.67(4.31)$ & $22.58(3.75)$ & $6.91(6.28$ to 7.55$)$ & $21.08(5)$ & $22.85(5.09)$ & 1.77 (0.9 to 2.62$)$ & $5.14(3.63$ to 6.65$)$ & 0.001 \\
\hline Strength lb. Hip flexors & $19.42(6.92)$ & $26.17(6.9)$ & $6.75(4.58$ to 8.91$)$ & $23.31(4.11)$ & $25.46(4.84)$ & $2.15(0.26$ to 4.04$)$ & $4.6(2.31$ to 6.89$)$ & 0.001 \\
\hline Hip abductor & $22.67(5.72)$ & $27.25(4.77)$ & $4.58(3.08$ to 8.7$)$ & $25.69(5.32)$ & $27.69(5.2)$ & $2(0.27$ to 3.72$)$ & $2.58(0.58$ to 4.58$)$ & 0.001 \\
\hline Hip adductor & $19.83(7.83)$ & $26.33(8.58)$ & $6.5(4.29$ to 8.7$)$ & $24(6.64)$ & $27.7(5.21)$ & $3.69(1.57$ to 4.88$)$ & $2.9(1.74$ to 6.56$)$ & 0.022 \\
\hline Gait speed, $\mathrm{m} / \mathrm{s}$ & $0.53(0.2)$ & $0.73(0.34)$ & $0.2(0.07$ to 0.3$)$ & $1.12(1.2)$ & $1.16(1.19)$ & 0.04 (0.01 to 0.07$)$ & $0.16(-0.1$ to 1.09$)$ & 0.006 \\
\hline Cadence, steps/min & $64.67(13.9)$ & $77.17(15.1)$ & $12.5(6.5$ to 18.48$)$ & $76.99(19.24)$ & $81.46(21.99)$ & $4.47(-2.4$ to 9.77$)$ & $8(-2.43$ to 12$)$ & 0.037 \\
\hline TIS 2.0 & $5.17(1.26)$ & $9.13(1.72)$ & $3.96(3.27$ to 5.05$)$ & $8.08(2.39)$ & $9.92(2.75)$ & $1.84(0.58$ to 2.95$)$ & $2.12(0.67$ to 3.57$)$ & 0.004 \\
\hline
\end{tabular}

FMA-LE, Fugl Meyer assessment for lower extremity; TIS 2.0, Trunk Impairment Scale version 2.0; MBI, Modified Barthel Index.

* Analyzed using paired $t$ test; ${ }^{\S}$ analyzed using independent $t$ test; ${ }^{\dagger} p$ value statistically significant and level of significance was set as $p<0.05$.

tional ability measured with MBI improved almost twofold in the experimental group (32\%), while it was $18 \%$ in the control group.

\section{Discussion}

The aim of this study was to determine the effects of pelvic stability training on the trunk and lower extremity movement control, hip muscles strength, walking speed and daily activities in patients with stroke. We found that pelvic stability training showed noteworthy improvements on the movement control of trunk and lower extremities, hip muscles strength, gait speed and lateral pelvic tilt except anterior pelvic tilt and MBI in patients with stroke.

The benefits of pelvic stability training were seen in the better trunk performance as measured by TIS 2.0. Biomechanically, the pelvis is a part of lower trunk in the sitting position providing dynamic postural stability during anterior-posterior and lateral weight shifts. To perform lateral flexion and rotation of trunk movements in sitting, an individual should have better dynamic stability of the lower trunk and pelvis. We observed a reduction in angle of lateral pelvic tilt (relative ratio of 1.8) post training. Patients after stroke typically show limited for- ward pelvic movement and inability to transfer body weight towards the most involved body portion - buttocks - in sitting [23]. A previous cross-sectional study reported a positive correlation between excessive lateral pelvic tilt in sitting and poor trunk performance in patients with stroke [24].

The observed 6.91 points mean changes in the FMALE scale favoring the pelvic stability training as compared to previously established minimal detectable change score of 3.57 points. We attribute this improvement to the proximal dynamic pelvic stability, which might have allowed for better intra-limb coordinated synergistic movements between hip, knee and ankle joints. Also, the selective activity of lower trunk and proximal hip muscles might have minimized the excessive co-contraction and stiffness of the most involved lower limb muscles, thereby possibly overcoming the stereotypic movement patterns [25]. The improvement in the hip extensor and abductor muscle strength could be related to the specificity of exercise regimen in which these muscles were trained for isometric contraction at their inner range against manual and mechanical resistance. We believe that the mean change of $2.8 \mathrm{lb}$. in the hip extensor strength supports the pelvic stability training. The change of $1.6 \mathrm{lb}$. in the hip extensor strength was recommended as a genuine change 
in the strength training regimen [26]. Due to the methodological limitations of a handheld dynamometer, we could not test the phasic isotonic contraction of hip flexors and adductors, but measured their isometric strength at certain joint angles. After pelvic stability training, the improvement in the isometric strength of these muscles might be related to more motor unit recruitment, re-organization of muscle fiber structure and increased rate of force development as an adaptive response [27, 28]. However, the use of isokinetic dynamometer in future trials shall provide a genuine change in the hip muscles strength following pelvic stability regime.

Increased gait speed with a mean difference of 0.16 $\mathrm{m} / \mathrm{s}$ could be related to better lower extremity motor control and hip muscle strength following 6 weeks of training. The change of gait speed observed in our study was similar to Milot et al. [29] who showed $0.14 \mathrm{~m} / \mathrm{s}$ change in post stroke household walkers after 6 weeks lower limb strength training. Pelvic movements are altered in stroke during walking and are related to gait speed $[8,30]$. An improved pelvic stability not only show a practice effect on balance ability but also allow better stance control and limb advancement, which are the major determinants of gait speed and cadence [31]. Our views are supported by Trueblood et al. [32] who demonstrated an immediate improvement on gait speed and cadence following PNF-based pelvic exercises. Enhanced forward propulsion of trunk segment and lateral pelvic displacement during stance phase are the other contributing factors for walking performance [33]. The repeated practice of stepping and weight transfer exercises in stride and step standing positions might have targeted the hip extensors and abductors activity. After stroke, the EMG activity of hip muscular activity during stepping closely resembles to healthy individuals [10]. The learning effect in response to exercise training might have improved the isometric activity of hip extensor during the stance phase, invariably allowing for peak hip and knee flexion momentum during the swing phase.

Overall, the mean change of 20 points and $32 \%$ improvement in the activities of daily living measured using MBI support the pelvic stability training. Pelvis being the functional kinetics of lower extremity while standing and during mobility, the proximal dynamic pelvic stability is essential to meet daily functional needs such as transfers, walking on the even floor and climbing upstairs. It is interesting to note that those who practiced standard physiotherapy care too improved (change of 12 points; 18\%) considerably in the physical function measure. Addressing from the clinical

Pelvic Stability Training on Movement Control and Daily Activities after Stroke perceptive of stroke recovery, even a minimal amount of change in activities of daily needs is clinically relevant. While interpreting the moderate dependence (MBI of 6190 points) at the pre-training level, both the groups progressing from severely moderate to slightly moderate dependency indicates lesser community support to deal with $[34,35]$. However, we are afraid that this notation should be confirmed in future interventional trials using the community-specific questionnaire as an outcome measure.

\section{Limitations and Future Implications}

The potential limitations of this study are that majority of study participants were males affected by ischemic stroke lesion with right-sided paralysis. Grouping of patients in regard to other co-morbidities was not performed due to the small sample size. Another potential limitation is that the strength of lower trunk muscles was not tested in this study. Nevertheless, the strength of lower limb muscles was not tested in the functional position; the isokinetic strength testing in future trials might give genuine effects of pelvic stability training. The study was conducted in a single rehabilitation setting and participants were not in the acute stage of stroke recovery. We believe that following them up with a longer study duration would further affirm the motor learning effects of pelvic stability training in patients with stroke. We further recommend the examination of the benefits of pelvic stability training on balance confidence and community participants in future clinical trials involving more number of study participants with follow-up.

\section{Conclusion}

Pelvic stability training is beneficial in improving the motor recovery of trunk and lower extremity, hip muscles strength, gait speed and activities of daily living in patients with stroke.

\section{Acknowledgement}

We are grateful to study participants.

\section{Disclosure Statement}

Authors disclose that there were no sponsorship or funding arrangements relating to our research and we also declare that there are no conflicts of interest to disclose pertaining to this study. 


\section{Authors Contribution}

L.D.: designing study, obtaining Ethics Committee approval, conducting interventions, handling outcome measures data, identifying, analyzing and interpreting data, writing and identifying relevant references. S.K.: designing study, conceptualized the pelvic stability regime, specifying the question, translating protocol into practice, identifying, analyzing and interpreting data, writing, reading, editing, and checking, identifying relevant references. D.M.: specifying the question, ensuring randomization process, analyzing and interpreting data, reading and checking.

\section{Appendix 1}

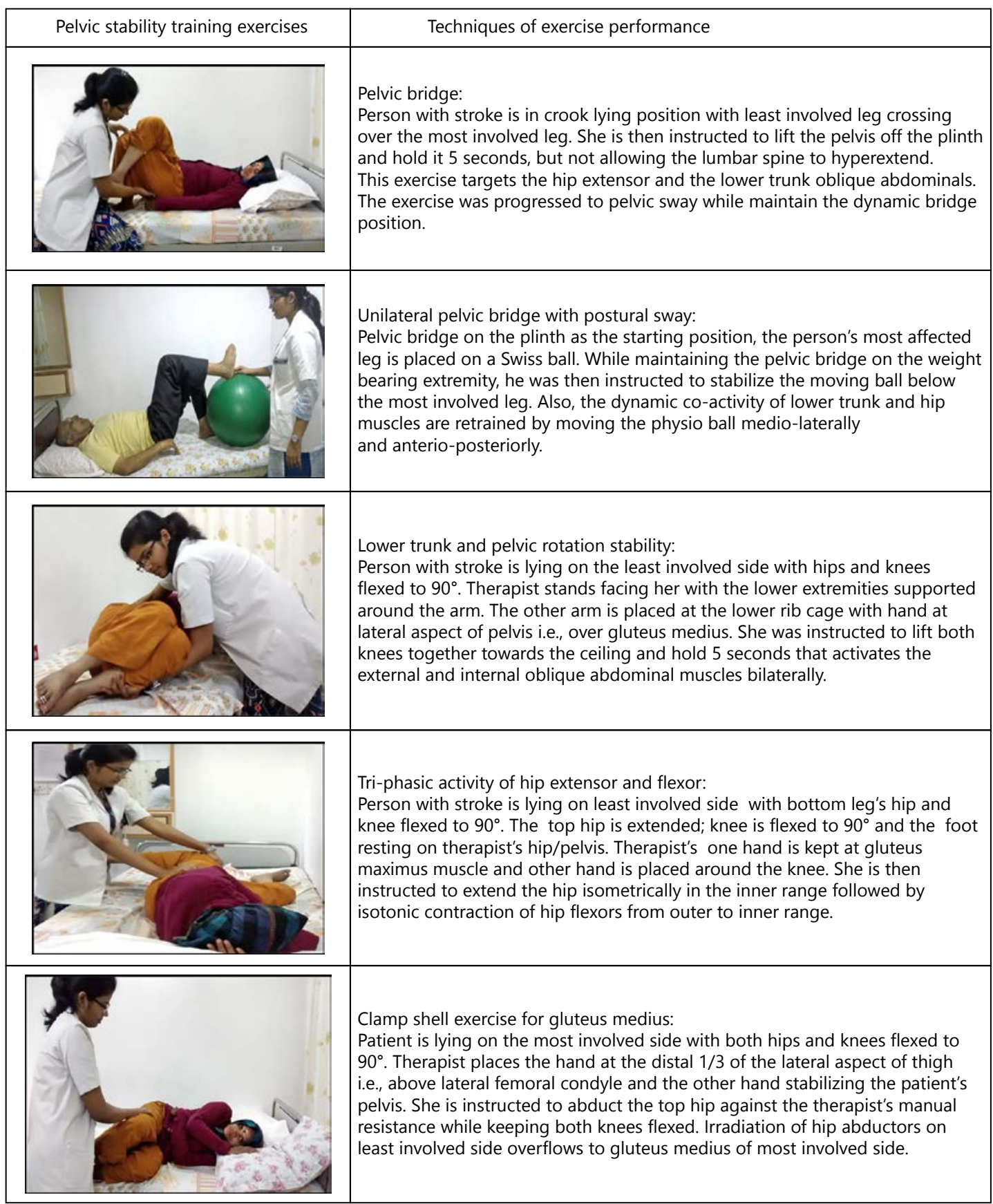




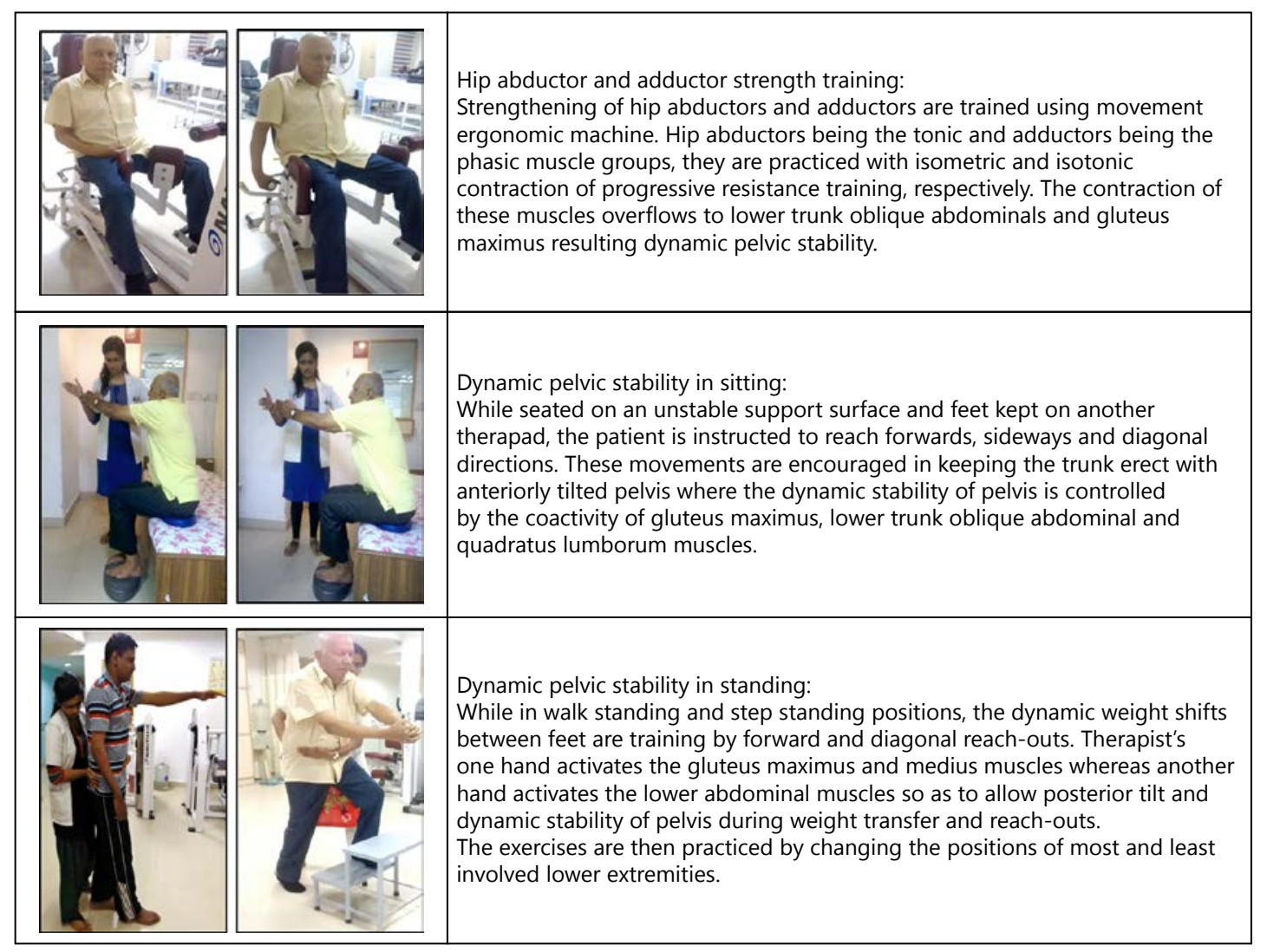

\section{Appendix 2}

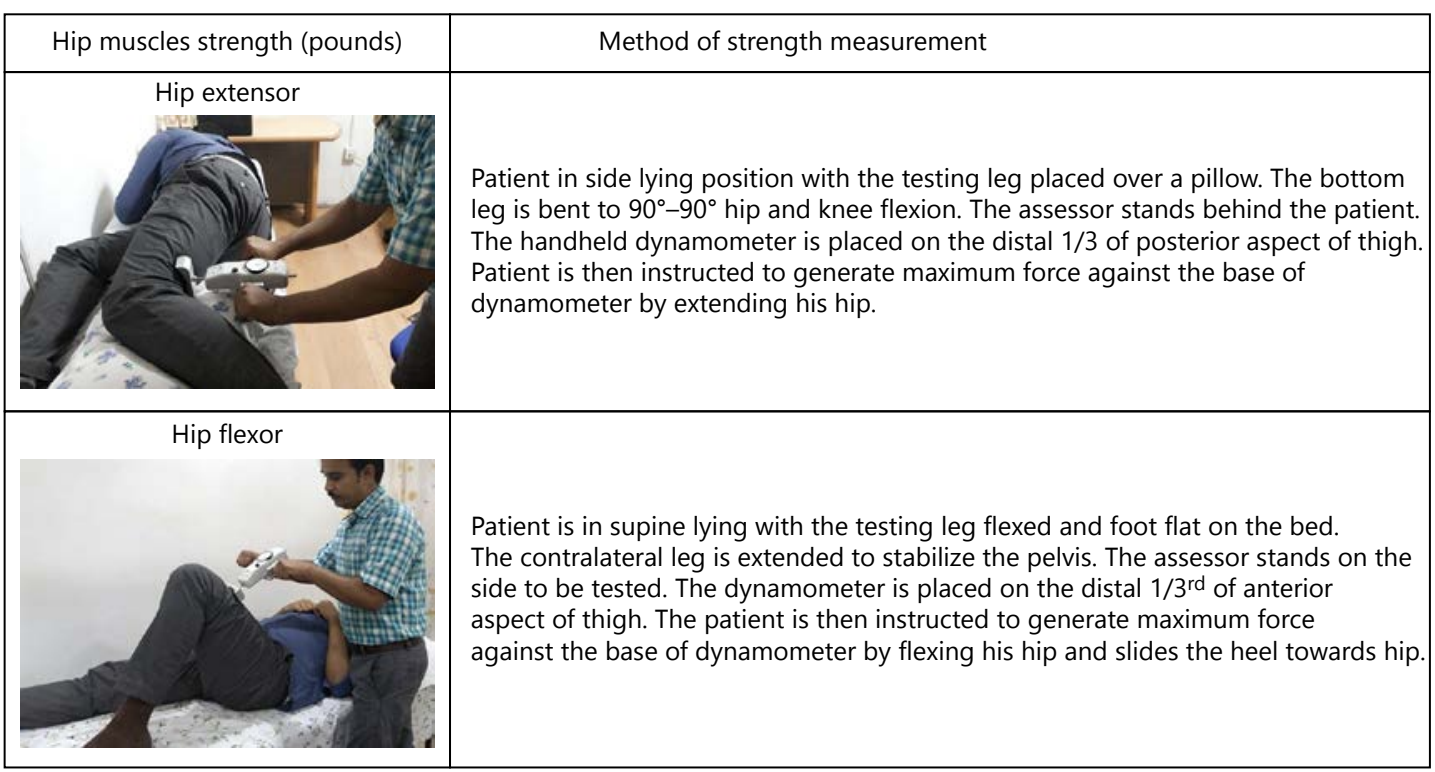

Pelvic Stability Training on Movement Control and Daily Activities after Stroke 


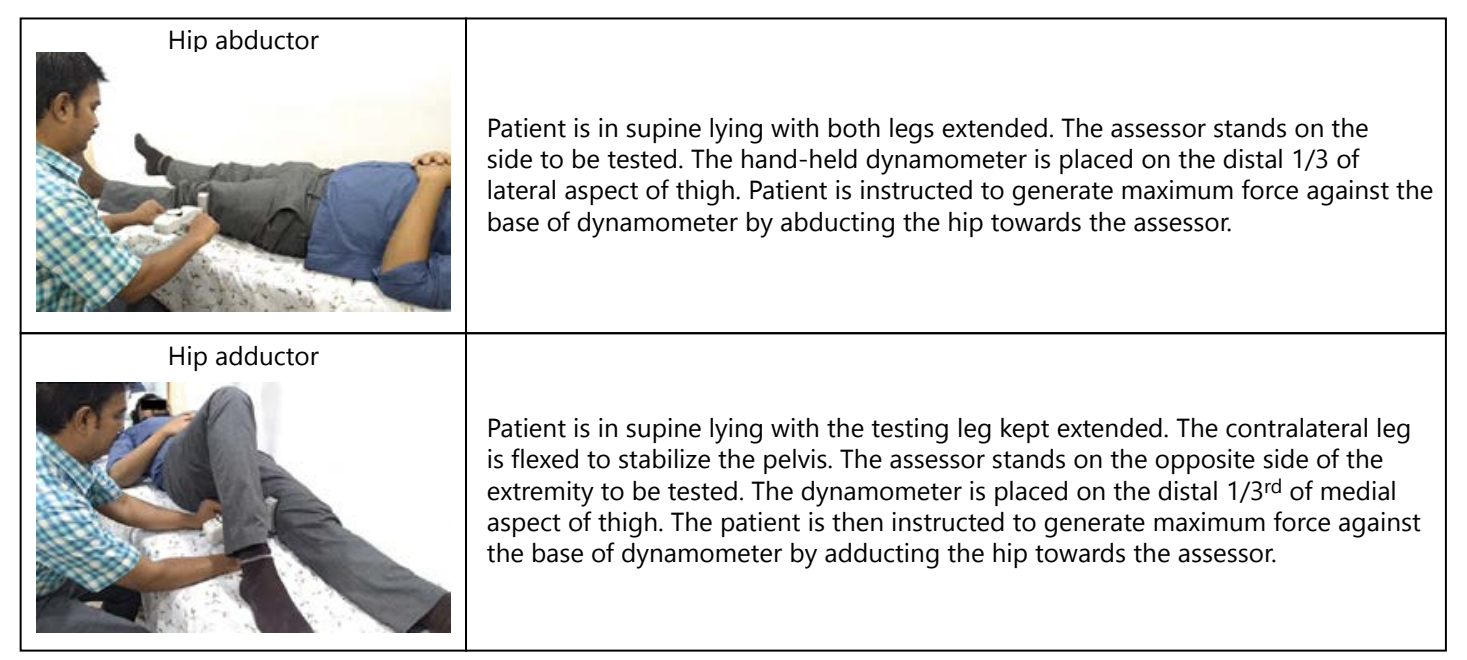

\section{References}

1 Danielsson A, Willen C, Sunnerhagen KS: Physical activity, ambulation, and motor impairment late after stroke. Stroke Res Treat 2012;2012:818513.

-2 Lawrence ES, Coshall C, Dundas R, Stewart J, Rudd AG, Howard R, et al: Estimates of the prevalence of acute stroke impairments and disability in a multiethnic population. Stroke 2001;32:1279-1284.

- 3 Garland SJ, Gray VL, Knorr S. Muscle activation patterns and postural control following stroke. Motor Control 2009;13:387411.

4 Lee D, Hodges P: Principles of integrated model of function and its application to the lumbopelvic- hip region; in Lee D, Hodges $\mathrm{P}$ (eds): The Pelvic Girdle, ed 3. Churchill Livingstone, 2004, pp 41-54.

5 Karthikbabu S, Chakrapani M, Ganesan S, Ellajosyla R: Pelvic alignment in standing, and its relationship with trunk control and motor recovery of lower limb after stroke. Neurol Clin Neurosci 2017;5:22-28.

-6 Verheyden G, Vereeck L, Truijen S, Troch M, Herregodts I, Lafosse C, et al: Trunk performance after stroke and the relationship with balance, gait and functional ability. Clin Rehabil 2006;20:451-458.

7 Karthikbabu S, Chakrapani M, Ganesan S, Ellajosyula R: Relationship between pelvic alignment and weight-bearing asymmetry in community-dwelling chronic stroke survivors. J Neurosci Rural Pract 2016;7(suppl 1):S37-S40.

8 Tyson SF: Trunk kinematics in hemiplegia gait and the effect of walking aids. Clin Rehabil 1999;13:295-300.

$\checkmark 9$ Genthon N, Rougier P, Gissot AS, Froger J, Pélissier J, Pérennou D. Contribution of each lower limb to upright standing in stroke patients. Stroke 2008;39:1793-1799.
10 Kirker SG, Simpson DS, Jenner JR, Wing AM: Stepping before standing: hip muscle function in stepping and standing balance after stroke. J Neurol Neurosurg Psychiatry 2000;8: 458-464.

11 Parvataneni K, Olney SJ, Brouwer B: Changes in muscle group work associated with changes in gait speed of persons with stroke. Clin Biomech 2007;22:813-820.

12 Dorsch S, Ada L, Canning CG: Lower limb strength is significantly impaired in all muscle groups in ambulatory people with chronic stroke: a cross-sectional study. Arch Phys Med Rehabil 2016;97:522-527.

13 Moore S, Schurr K, Wales A, Moseley A, Herbert R: Observation and analysis of hemiplegic gait: swing phase. Aust J Physiother 1993; 39:271-278.

14 Cruz TH, Lewek MD, Dhaher YY: Biomechanical impairments and gait adaptations post-stroke: multi-factorial associations. J Biomech 2009;42:1673-1677.

15 Verheyden G, Kersten P: Investigating the internal validity of the Trunk Impairment Scale (TIS) using rasch analysis: the TIS 2.0. Disabil Rehabil 2010;32:2127-2137.

-16 Sullivan KJ, Tilson JK, Cen SY, Rose DK, Hershberg J, Correa A, et al: Fugl-Meyer assessment of sensorimotor function after stroke: standardized training procedure for clinical practice and clinical trials. Stroke 2011;42: 427-432.

17 Shelton FD, Volpe BT, Reding M: Motor impairment as a predictor of functional recovery and guide to rehabilitation treatment after stroke. Neurorehabil Neural Repair 2001;15: 229-237.

18 Karthikbabu S, Chakrapani M: Hand-held dynamometer is a reliable tool to measure trunk muscle strength in chronic stroke. J Clin Diagn Res 2017;11:YC09-YC12.
19 Martins JC, Aguiar LT, Lara EM, TeixeiraSalmela LF, Faria CD. Assessment of the strength of the lower limb muscles in subjects with stroke with portable dynamometry: a literature review. Physiother Mov 2016;29:193208.

20 Dacruz SN, KarthikBabu S, Syed N: Intra-rater and inter-rater reliability of measuring pelvic tilt using the palpation meter (PALM TM) device in stroke. Int J Med Health Sci 2016;5: 276-280.

21 Fulk GD, Ludwig M, Dunning K, Golden S, Boyne P, West T: Estimating clinically important change in gait speed in people with stroke undergoing outpatient rehabilitation. J Neurol Phys Ther 2011;35:82-89.

22 Hsueh IP, Lee MM, Hsieh CL: Psychometric characteristics of the Barthel activities of daily living Index in stroke patients. J Formos Med Assoc 2001; 100:526-532.

23 Messier S, Bourbonnais D, Desrosiers J, Roy Y: Dynamic analysis of trunk flexion after stroke. Arch Phys Med Rehabil 2004;85: 1619-1624.

24 Pathak S, Kumar V, Nayak A, Kedambadi R: The relationship between pelvic alignment and trunk control in stroke subjects: a crosssectional study. Int J Res Med Sci 2014: 2:1483-1487.

25 Hiengkaew V, Jitaree K, Chaiyawat P: Minimal detectable changes of the Berg Balance Scale, Fugl-Meyer assessment scale, timed "up \& go" test, gait speeds, and 2-minute walk test in individuals with chronic stroke with different degrees of ankle plantarflexor tone. Arch Phys Med Rehabil 2012;93:12011208.

26 Lee SB, Kang KY: The effects of isokinetic eccentric resistance exercise for the hip joint on functional gait of stroke patient. J Phys Ther Sci 2013;25:1177-1179. 
27 Freire B, Dias CP, Oliveira LS, Goulart NBA, Lemos FA, Becker J, et al: Rate of force development and torque production assessment in spastic stroke survivors. Brazil J Kinanthropometry Human Perform 2015;17:328336.

28 Eng JJ: Strength training in individuals with stroke. Physiother Can 2004;56:189-201.

-29 Milot MH, Nadeau S, Gravel D, Bourbonnais D: Gait performance and lower-limb muscle strength improved in both upper-limb and lower-limb isokinetic training programs in individuals with chronic stroke. ISRN Rehabil 2013;1-10.
0 Kong SW, Jeong YW, Kim JY: Correlation between balance and gait according to pelvic displacement in stroke patients. J Phys Ther Sci 2015;27:2171-2174.

31 Park BS, Kim JH, Kim MY, Lee LK, Yang SM, Jeon HJ, et al: Effect of a muscle strengthening exercise program for pelvic control on gait function of stroke patients. J Phys Ther Sci 2015;27:641-644.

32 Trueblood PR, Walker JM, Perry J, Gronley JK: Pelvic exercise and gait in hemiplegia. Phys Ther 1989;69:18-26.
33 Ceccato JC, de Sèze M, Azevedo C, Cazalets JR: Comparison of trunk activity during gait initiation and walking in humans. PLoS One 2009; 4:e8193.

34 Van Exel NJ, Scholte op Reimer WJ, Koopmanschap MA: Assessment of post-stroke quality of life in cost-effectiveness studies: the usefulness of the Barthel Index and the EuroQOL-5D. Qual Life Res 2004;13:427-433.

35 Duffy L, Gajree S, Langhorne P, Stott DJ, Quinn TJ: Reliability (inter-rater agreement) of the Barthel Index for assessment of stroke survivors: systematic review and meta-analysis. Stroke 2013;44:462-468. 